\title{
Research
}

\section{Spatial scale, topography and thermoregulatory behaviour interact when modelling species' thermal niches}

\author{
Madeleine G. Barton, Susana Clusella-Trullas and John S. Terblanche
}

M. G. Barton (http://orcid.org/0000-0001-9242-0800) (mbarton01@hotmail.com) and J. S. Terblanche, Centre for Invasion Biology, Dept of Conservation Ecology and Entomology, Stellenbosch Univ., South Africa. - S. Clusella-Trullas, Centre for Invasion Biology, Dept of Botany and Zoology, Stellenbosch Univ., South Africa.

\section{Ecography}

42: 376-389, 2019

doi: $10.1111 /$ ecog. 03655

Subject Editor: Sarah Diamond Editor-in-Chief: Miguel Araújo Accepted 3 June 2018
The spatial scale at which climate and species' occupancy data are gathered, and the resolution at which ecological models are run, can strongly influence predictions of species performance and distributions. Running model simulations at coarse rather than fine spatial resolutions, for example, can determine if a model accurately predicts the distribution of a species. The impacts of spatial scale on a model's accuracy are particularly pronounced across mountainous terrain. Understanding how these discrepancies arise requires a modelling approach in which the underlying processes that determine a species' distribution are explicitly described. Here we use a process-based model to explore how spatial resolution, topography and behaviour alter predictions of a species thermal niche, which in turn constrains its survival and geographic distribution. The model incorporates biophysical equations to predict the operative temperature $\left(\mathrm{T}_{e}\right)$, thermal-dependent performance and survival of a typical insect, with a complex life-cycle, in its microclimate. We run this model with geographic data from a mountainous terrain in South Africa using climate data at three spatial resolutions. We also explore how behavioural thermoregulation affects predictions of a species performance and survival by allowing the animal to select the optimum thermal location within each coarse-grid cell. At the regional level, coarse-resolution models predicted lower $\mathrm{T}_{e}$ at low elevations and higher $\mathrm{T}_{e}$ at high elevations than models run at fineresolutions. These differences were more prominent on steep, north-facing slopes. The discrepancies in $T_{e}$ in turn affected estimates of the species thermal niche. The modelling framework revealed how spatial resolution and topography influence predictions of species distribution models, including the potential impacts of climate change. These systematic biases must be accounted for when interpreting the outputs of future modelling studies, particularly when species distributions are predicted to shift from uniform to topographically heterogeneous landscapes.

Keywords: microclimate, spatial resolution, topography

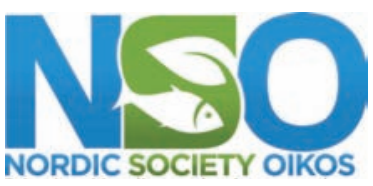

@ 2018 The Authors. Ecography ( 2018 Nordic Society Oikos

www.ecography.org 


\section{Introduction}

A major challenge of climate change research is to develop accurate means of predicting species distributions and persistence. Despite several advances in species distribution models (SDMs), a number of limitations still need to be addressed to improve their accuracy and broader applicability (Guisan and Thuiller 2005, Elith and Leathwick 2009, Levy et al. 2014). Both correlative and mechanistic modeling approaches are, at least to some extent, limited by spatial resolution due to the scale at which organism distribution and climatic datasets are currently available (Flint and Flint 2012, Beck et al. 2014). Many models, for example, are simulated across continental regions, with a resolution of 30 arc-seconds (approximately $1 \mathrm{~km}^{2}$ ), a reflection of the most readily available Geographical Information Systems (GIS) datasets. While this spatial resolution might be appropriate for relatively large and mobile organisms, average climactic conditions within large grid cells might misrepresent conditions encountered by small, less mobile species (Helmuth et al. 2006, Potter et al. 2013, Nadeau et al. 2017). The general ecology of the species in question, (e.g. endothermy vs ectothermy, body size, home range and mobility) and the spatial resolution of simulations can greatly influence the accuracy of the model's predictions and management recommendations based thereon (Guisan and Thuiller 2005, Seo et al. 2009).

Assigning an appropriate spatial scale to a SDM is particularly important across heterogeneous terrain where elevation, slope, aspect, and thus local microclimates, can change markedly over small distances (Scherrer and Körner 2011, Suggitt et al. 2011). Several studies have characterized variation in microclimates between adjacent sites that vary only in slope and aspect, typically finding substantial differences in multiple microclimatic parameters. Bennie et al. (2008), for example, showed that temperatures on sunlit slopes exceeded ambient temperature by up to $14^{\circ} \mathrm{C}$, while temperatures on shaded aspects remained close to ambient conditions. Such variability in temperature is comparable to broad-scale thermal variation along latitudinal and altitudinal gradients, and far exceeds changes in temperature that are predicted to occur under future climate warming scenarios (Billings 1973, Suggitt et al. 2011). When SDMs are developed from coarse resolution datasets of climatic variables, often averaged within each grid, these variables will 1) rarely represent the environments actually encountered by organisms (Weiss et al. 1988, Potter et al. 2013); 2) fail to capture thermal variability, and 3) overlook potential refuges within which populations might persist (Suggitt et al. 2011, Gillingham et al. 2012, Hannah et al. 2014). In light of these limitations, there has been a recent call for ecologists to not only re-address the scales at which underlying data are collated and models are run, but also to include topographic parameters (e.g. rugged versus flat terrain) when predicting species survival and performance across the landscape (Luoto and Heikkinen 2008, Austin and Van Niel 2010, Potter et al. 2013, Slavich et al. 2014, Nadeau et al. 2017). This pressing need for more detailed modelling frameworks is particularly dire for studies across mountainous regions, into which species distributions are frequently shifting, where weather data on different slopes are less frequently collected, and where highly specialized montane species are increasingly stressed (Parmesan and Yohe 2003, Chen et al. 2011, Nieto-Sanchez et al. 2015).

While the importance of assigning an appropriate spatial scale in SDMs has been emphasized previously (Guisan and Thuiller 2005, Araújo and Guisan 2006), understanding the direction and extent to which scale affects predictions remains unclear (Guisan et al. 2007, Seo et al. 2009). For example, SDMs of alpine plant responses to climate change produced from coarse resolutions generated both smaller and larger predicted distributions in comparison to models run at relatively finer resolutions (Trivedi et al. 2008, Randin et al. 2009). A subsequent study found that a finer resolution led to an increase in the predicted distributions of 52 plant species in California; however, simulations with projected climate warming scenarios generated inconsistent shifts between spatial scales and among species (Franklin et al. 2013). Similarly, Gillingham et al. (2012) found that increasing spatial resolution resulted in greater model accuracy but the impacts of climate change on predicted distributions of species did not vary in a systematic manner among resolutions. Thus, it remains unclear whether systematic, predictable errors exist when scaling up or down between resolutions when forecasting impacts, or whether the contrasting findings of these studies are context-dependent. Consequently, these results add uncertainty, and perhaps even significant errors, when interpreting SDM outputs for climate change forecasting, thereby raising the issue of model validity and transferability of results among spatial scales and terrestrial environments.

The studies described above have predominantly focused on plant species (though see Gillingham et al. 2012), with the assumption that individuals are constrained in space with limited mobility and little capacity for behavioural thermoregulation. For mobile organisms with the capacity to behaviourally buffer environmental stress however, the accuracy of species distribution models (both correlative and mechanistic) are improved when behavioural traits, such as site selection, are incorporated into the modelling framework (Buckley et al. 2013, 2015). As such, discrepancies between the outcomes of models run on different resolutions might be further affected if the organism has the ability to move across landscapes, to actively select preferred microclimates. Indeed, across a typical grid matrix used for modelling studies (cells of $1 \mathrm{~km}^{2}$ ), animals can shift across small distances within each cell, for example between vegetation and below ground refugia or across larger distances between grid-cells (Kearney et al. 2009, Ruiz-Aravena et al. 2014, Woods et al. 2015). Depending on the mobility of the animal, opportunities to behaviourally thermoregulate are greatest in habitats with high levels of thermal heterogeneity (Slavich et al. 2014, Sears et al. 2016, Basson et al. 2017), such as those encountered in mountainous regions (Hertz et al. 1993, Deutsch et al. 2008, Bonebrake et al. 2014, Hannah et al. 2014, Lawson et al. 2014). When SDMs are run at coarse resolutions, models might fail to capture fine-scale variation 
in not only microclimates and their variability, but also the animal's capacity to exploit thermal heterogeneity and optimize performance.

Moreover, many of these studies described above employ a correlative modelling approach whereby the effects of climate and topography on organism survival are implicitly captured in the species presence/absence datasets (Trivedi et al. 2008, Randin et al. 2009, Franklin et al. 2013). While Gillingham et al. (2012) incorporate outputs of a microclimate model as predictor variables in their correlative SDM, their final outputs predict only the the occupancy (presence/ absence) of the organism within a given grid cell. Despite the consideration of fine scale variation in microclimates in these models, the correlative approach provides minimal insights into the processes by which the discrepancies in the predictions between different spatial resolutions arise. Biophysical models on the other hand explicitly define how topographic features of a landscape directly affect operative temperatures ( $\mathrm{T}$ e hereafter), and subsequently, the performance and survival of the organisms within them. While mechanistic models often over-predict a species distribution (i.e. by predicting the fundamental rather than the realized niche; Kearney and Porter 2004), translating information of the physical environment (in this case temperature) into biologically relevant traits of the organism provides more informative outputs than a habitat suitability prediction alone (Buckley et al. 2010). Moreover, the physical equations, which explicitly define the interactions between an organism and its immediate environment, can be used to isolate any systematic changes in predicted microclimates, resulting $\mathrm{T}_{e}$, and species distributions between models run at different spatial resolutions. Explicitly predicting the $\mathrm{T}_{e}$ of organisms within their microclimates also enables testing a number of additional questions as to how the assigned scale might overlook detailed climate-organism interactions across different types of terrain (e.g. rugged vs smooth; Sears et al. 2011). For example, one can test the extent to which behavioural thermoregulation of mobile organisms within a grid cell might alter a model's predictions (Lawson et al. 2014, Woods et al. 2015).

Here, we use a mechanistic model to estimate the $\mathrm{T}_{e}$ of a lepidopteran species within a typical microclimate, and then test how spatial resolution, topography and behavioural thermoregulation affect predictions of $\mathrm{T}_{e}$, performance of temperature-sensitive traits and mortality across a rugged landscape (Buckley et al. 2010). A biophysical model has previously been developed for the African bollworm Helicoverpa armigera (Lepidoptera: Noctuidae) (Barton and Terblanche 2014), but here we present it as a general model for an insect with a complex life-cycle (holometabolous) that can complete multiple generations per year (multivoltine). The model was run on hourly time-steps tracking the animal as it matures and transitions through multiple life-history stages and generations, based on the predicted $\mathrm{T}_{e}$. Simulations were conducted at three spatial resolutions using historical monthly averages for a region in the Western Cape Province, South Africa, across which topography varies substantially (Fig. 1d). With these simulations we undertook a sensitivity analysis, testing: 1) how spatial resolution affects predictions of $T_{e}$, the number of generations completed as a proxy for fitness, and the thermal safety margin as a measure of thermal suitability (Deutsch et al. 2008), 2) if variation in the predicted $\mathrm{T}_{e}$ between scales is consistent with particular features of the landscape, and 3) the extent to which an animal's thermoregulatory behaviour might alter predictions of its fitness. We then discuss our results in the context of future climate change.

\section{Material and methods}

\section{Climate and topographical data}

The recently published microclimate model (Barton and Terblanche 2014; and Supplementary material Appendix 1) used in this study translates spatially explicit climatic datasets

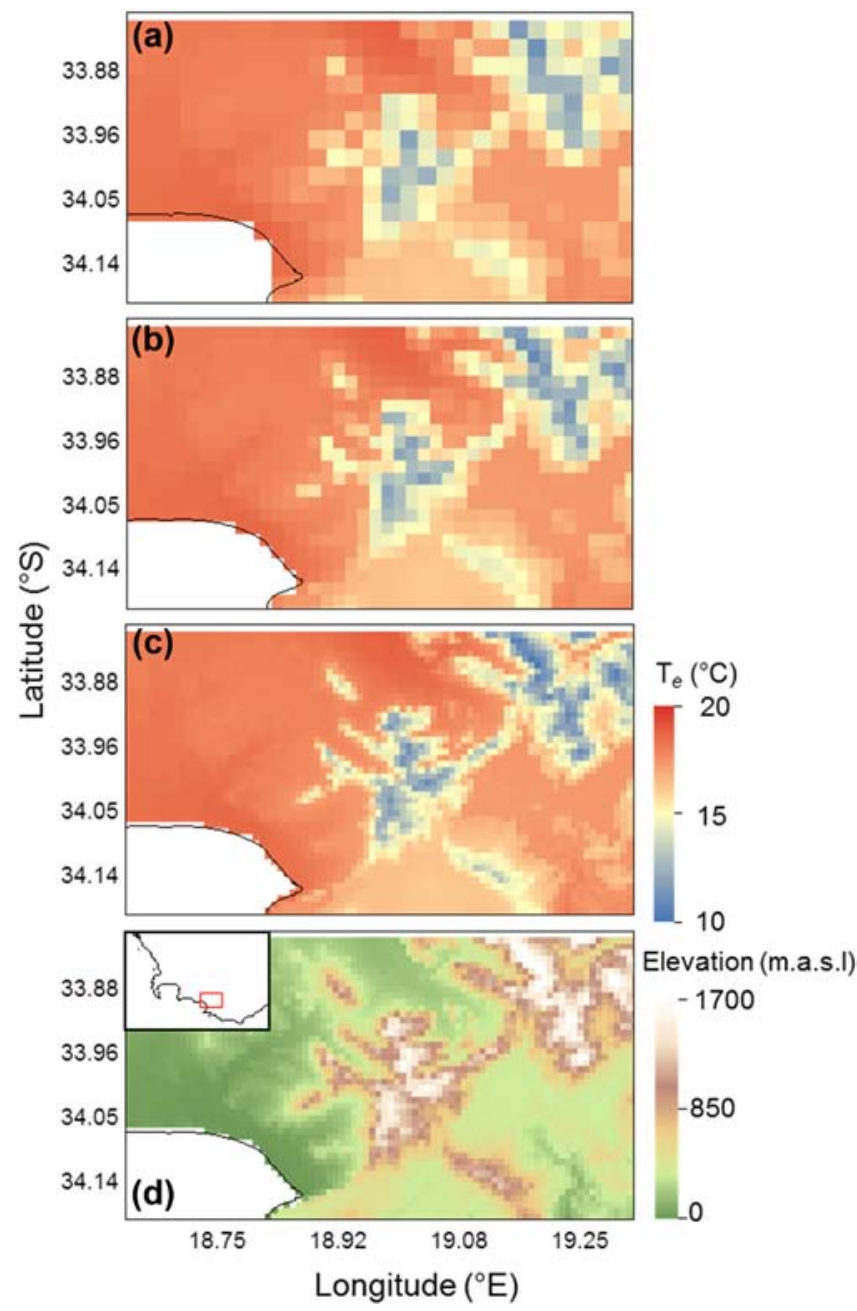

Figure 1. Predictions of mean annual operative temperature $\left(\mathrm{T}_{e}\right.$, in ${ }^{\circ} \mathrm{C}$ ) of a typical lepidopteran species modelled at (a) coarse, (b) medium and (c) fine resolutions. To obtain $\mathrm{T}_{e}$, all life-history stages were considered. Geographic extent encompasses a fruit growing region of the Western Cape Province in South Africa (inset), across which elevation ranges from 1 to in excess of $1700 \mathrm{~m}$ a.s.l. (d). 
into the microclimatic conditions experienced by the organism. More specifically, the model predicts the $\mathrm{T}_{e}$ of a typical holometabolous insect (Helicoverpa armigera) using a set of biophysical equations and accounts for the different lifehistory stages occupying different sites within the habitat (i.e. eggs and larvae are located on a leaf in a tree canopy, pupae underground, and adults on the surface of a tree trunk). While we have populated the model with thermal sensitivity data from of $H$. armigera as an example, alternative datasets for a species of interest could be easily substituted if desired. For this study, we expand upon several aspects of the earlier model to address our study aims. Specifically, here climatic and elevation data incorporated into this model were sourced at 30 arc-second (approximately $1 \mathrm{~km}^{2}$ ) cell resolution from the Worldclim database, ver. 1.4 (Hijmans et al. 2005; <www.worldclim.org/current.htm>) for monthly averages from 1960-1990, interpolated across an agricultural region of the Western Cape, South Africa (extent: $33.82-34.22^{\circ} \mathrm{S}$, $18.63-19.36^{\circ} \mathrm{E}$, Fig. 1d). Maximum temperature, minimum temperature, and relative humidity (not available in Worldclim, and thus sourced from Schulze 1997), as well as elevation were required as input data. Furthermore, in this study, to generate measures of temperature at hourly timesteps, we first splined the monthly records of maximum and minimum temperatures into 365 daily data-points, which were then used in an interpolation of a daily sinusoidal wave according to Campbell and Norman (1998). Topographic characteristics across the landscape were attained using the 'slope/aspect' functions under the 'raster' package written for $\mathrm{R}$ (ver. 3.1.2, all analyses hereafter, Hijmans and van Etten 2012) that compares the elevation of adjacent cells to compute average slope and aspect of each grid cell. An additional calculation was included in the model (in comparison to that published in Barton and Terblanche 2014), in which hill-shading at a given location from adjacent sites was determined by comparing the horizon angle in the direction of the sun's azimuth (with the 'horizon' package, Van doninck 2016) against the sun's height in the sky (90-zenith) at each hour of the simulation (Supplementary material Appendix 1). If the corresponding horizon angle was higher than the sun's elevation, we assumed that direct solar radiation, and any heat gained by the animal through this source, was negligible.

These raw climatic and topographic datasets were considered to be fine-scale, with a total of 4043 grid cells. Using these data we generated a medium resolution with 1033 sites (60 arc-seconds, $\sim 2 \mathrm{~km}^{2}$ ) and a coarse resolution with 466 sites (90 arc-seconds, $\sim 3 \mathrm{~km}^{2}$ ) input dataset using the aggregate() function in the 'raster' package. This function calculates the mean values for elevation and each climatic variable across 4 and 9 fine-grid cells for the medium and coarse resolutions respectively, where the top-left hand cell remains aligned in all resolutions (the same approach taken to generate the coarse resolution datasets for the Worldclim database; <www.worldclim.org/formats1>; Supplementary material Appendix 2 Fig. A1). From the medium and coarse elevation datasets, aspect and slope were subsequently calculated using the 'raster' package (see previous paragraph). These resulting fine, medium and coarse resolution datasets of climate and topography were each used as inputs in the model to predict the insect's $\mathrm{T}_{e}$ (Bakken 1992 processes defined in detail in Supplementary material Appendix 1). We consider these fine, medium and coarse resolutions to be at the scale of a typical lepidopteran's home range or expected core population occupancy (King et al. 1990). Finer resolution climatic datasets for the region of interest are unfortunately unavailable and the computational time involved becomes extremely long. Finally, here we assume that $T_{e}$ equates to the insect's body temperature, due to its small size, high thermal conductance and lack of physiological thermoregulatory mechanisms (Stevenson 1985).

\section{Model simulations}

For each site, at each hour of these simulations, the predicted $\mathrm{T}_{e}$ was used to calculate 1) the developmental progression as the number of degree-day units attained $\left(\mathrm{DD}=\mathrm{T}_{\mathrm{e}}-\mathrm{T}_{\min }\right)$ where $\mathrm{T}_{\min }$ is $H$. armigera's lower developmental threshold for each stage (Supplementary material Appendix 2 Table A1) and 2) the thermal safety margin that compares the predicted $\mathrm{T}_{e}$ of the insect against its optimum temperature $\left(\mathrm{TSM}=\mathrm{T}_{\mathrm{opt}}-\mathrm{T}_{\mathrm{e}}\right)$ where $\mathrm{T}_{\mathrm{opt}}$ is $27.5^{\circ} \mathrm{C}$, the optimum temperature for population growth (Mironidis 2014). The TSM formula follows Deutsch et al. 2008 and differs from that defined by Sunday et al. 2014, who used TSM as the difference between the species $\mathrm{CT}_{\max }$, and the highest predicted $\mathrm{T}_{e}$. As the simulations cycled through progressive time steps, the total numbers of degree-day units were tallied and the model transitioned through successive life-history stages. The thresholds for degree-day units were multiplied by 24 , to account for the hourly time intervals of the model. Once the adult stage was reached, the model remained in the adult microclimate for an additional 100 degree-day units (ranging from 4 to $36 \mathrm{~h}$ depending on the location and season), after which the model reverted back to the egg stage, as female adults are typically able to lay eggs soon after eclosion (Mironidis 2014). At this transitional hour, the accumulated degree-days were reset to zero and development as an egg re-commenced. At each site across the landscape the number of completed generations (referred to as voltinism hereafter) through the entire simulation period $\left(8760 \mathrm{~h}\right.$ in total), and the average $\mathrm{T}_{e}$ and TSM were determined.

\section{Defining the thermal niche}

To understand how the model resolution affects predictions of a species thermal niche, widely considered to be an important underlying process that constrains the survival and distribution of terrestrial ectotherms (Kearney and Porter 2004, Wiens et al. 2010), we set critical thermal limits to simulate a cold- and a heat-sensitive species. For these simulations, we assumed that the animal could not survive 
if $\mathrm{T}$ exceeded thermal critical limits, i.e. the critical thermal minimum $\left(\mathrm{CT}_{\min }\right)$ of the cold sensitive species was reduced to $8^{\circ} \mathrm{C}$, while the critical thermal maximum $\left(\mathrm{CT}_{\max }\right)$ for the heat sensitive species was held at $40^{\circ} \mathrm{C}$ (in comparison to the original $H$. armigea thermal tolerance measures; Mironidis and Savopoulou-Soultani 2008). If, at any hour during the simulation period, $T_{e}$ exceeded these thresholds, the organism (or population) was assumed to perish and be absent from that grid cell. These conditions were applied to the fine-, medium- and coarse-resolution $\mathrm{T}_{e}$ outputs, and the resulting thermal niches, which included occupied cells only, were subsequently mapped.

\section{Behaviour within grid cells}

We explored how incorporating behavioural thermoregulation affects model predictions by allowing the animal to seek out and occupy thermally optimal sites, assuming their home-range is the size of a coarse grid cell in our simulations $\left(3 \mathrm{~km}^{2}\right.$ is a typical home-range size in the absence of long-range dispersal events; King et al. 1990). We assumed that only the adult stage is sufficiently mobile to select optimum locations within this home range, and re-ran the coarse-scale simulation but this time, when the model transitioned into the adult phase, the most optimal finescale grid cell, within which temperature was closest to the adult's thermal optimum of $27.5^{\circ} \mathrm{C}$ (Rosenheim et al. 2008, Bonebrake et al. 2010, Mironidis 2014), was selected. On the first hour when the model transitioned back to the egg stage, conditions within the adult's selected location (1 of the 9 fine grid cells) were used to represent the entire homerange (the coarse-grid cell). In the subsequent hours of the egg, larval and pupal stages, the entire home-range continued to reflect the changing hourly climatic conditions (and $\mathrm{T}_{e}$ ) within this location. Upon reaching the adult stage once more (the following generation), the organism reselected the most optimal location within the coarse-grid cell, thereby once again dictating the subsequent conditions experienced by the offspring. This procedure was repeated for every generation. Ultimately, with this simulation we aim to explicitly test the extent to which female adult thermoregulatory behaviour during oviposition might affect the survival and performance of their offspring.

We then compared the predicted voltinism of this 'thermoregulator' simulation with those measures of the previous coarse-model predictions considered as 'sessile' (i.e. no thermoregulation) as a measure of the impacts of behaviour on physiological performance. To ascertain how such behaviour might buffer extreme temperatures and thus survival, we also compared the total number of hours during which extreme cold- and hot-conditions were encountered by the 'thermoregulator' and 'sessile' organism. In this case, we assumed extreme-stress (and ultimately death) occurred when $\mathrm{T}_{\mathrm{e}}<\mathrm{CT}_{\min }$ or $\mathrm{T}_{\mathrm{e}}>\mathrm{CT}_{\max }$, where $\mathrm{CT}_{\min }$ and $\mathrm{CT}_{\max }$ are $8^{\circ} \mathrm{C}$ and $40^{\circ} \mathrm{C}$ respectively (Mironidis and SavopoulouSoultani 2008).

\section{Statistical analysis}

To understand how resolution affected our predictions of the insect's T, TSM and voltinism (response variables hereafter) across the Western Cape Province, the coarse and medium resolution output datasets were disaggregated to the fine-scale resolution grid, such that each simulation output comprised of the same number of sites $(n=4043)$. In analyses, aspect was calculated in terms of deviations away from due north (the sun's azimuth at noon in the southern hemisphere), making the assumption that northerly facing sites would, in general, receive higher loads of direct solar radiation than slopes with southern aspects.

We built a series of generalized linear mixed effects models (GLMMs) for each response variable, to explore how topographic features across the modelling extent affected the model's predictions as a function of scale (Zuur et al. 2009). These 'full' models each included interactions between elevation, slope, aspect, and scale (coarse or fine resolution), as well as elevation to the second polynomial term, as fixed effects. Preliminary statistics indicated that there were no substantial differences in predictions between the medium and fine-resolution datasets, and so for simplicity, these analyses considered only data from the coarse and fine-scale resolutions. Site (or location/pixel) was included as a random factor to account for pseudo replication among the two spatial scales (each site was represented two times). Predictions of $\mathrm{T}_{e}$ and TSM were positively skewed (with relatively more sites at lower elevations), and thus a Gamma log-likelihood distribution was assigned to the GLMMs, while that for voltinism was modelled with a Poisson distribution.

To account for non-independence of errors due to spatial auto-correlation among the data, we also incorporated an auto-covariate term, calculated with the 'autocov_dist' function with the 'spdep' package in R (Dormann et al. 2007, Bivand and Piras 2015). This covariate is weighted by the square of the inverse distance among neighbourhood cells (set to a distance of 15 grid cells).

Starting with the full model for each response variable (described above), we sequentially removed interaction and polynomial parameter terms until the ('reduced') model of best fit was attained, determined by the BIC value (and where $\triangle \mathrm{BIC}>2$ ), deemed to be a more conservative criterion than AIC for models with large sample sizes and a relatively high number of predictor variables (Acquah 2010). Multi-collinearity among input variables of the reduced models was confirmed to not be a confounding effect, with all variance inflation factors below the accepted threshold of 10 (Neter et al. 1996, Zuur et al. 2009). With the reduced model for each response variable, we then generated semivariograms of the normalized residuals to confirm that there were no confounding effects due to spatial auto-correlation. The GLMMs, model comparisons, and semi-variogram analyses were conducted in R with the 'Ime4' (Pinheiro et al. 2016), 'MuMIn' (Barton 2016), and 'gstat' (Gräler et al. 2016) packages respectively. 
The predicted coarse, medium and fine resolution thermal niches (the number of occupied grid cells) for both the cold and heat sensitive species were compared using a Friedman rank sum test, with site included as a random factor. Finally, to determine if dispersal of the female adult to an optimum thermal location within each coarse grid cell had significant impacts on our predictions of thermal stress and voltinism, we ran paired t-tests comparing the 'thermoregulating' and 'sessile' model predictions for each trait. Given the large sample size in these analyses $(n=466$, the coarse grid), we set $\mathrm{p}<0.001$ as the threshold level for significance.

\section{Data deposition}

Code for the model (R scripts) and analyses, as well as raw output files from the model, are available from the authors on request.

\section{Results}

\section{Regional simulations}

The predicted annual mean $T_{e}$ of the insect varied spatially across the simulated region of the Western Cape. Predictions of $\mathrm{T}$ e from the fine and medium resolution simulations ranged from approximately $10^{\circ} \mathrm{C}$ to $19^{\circ} \mathrm{C}$ (Fig. $1 \mathrm{~b}$, c), while the coarse resolution model predicted $\mathrm{T}_{e}$ from $11^{\circ} \mathrm{C}$ to $18^{\circ} \mathrm{C}$ (Fig. 1a). As elevation increased, $\mathrm{T}_{e}$ declined, and was significantly colder on shallow, southerly facing slopes as opposed to steep sites with northern aspects (Fig. 1, Table 1). These predictions of $\mathrm{T}_{e}$ however varied among the fine and coarse spatial scales, depending on the elevation, slope and aspect of each site (Table 1).

To visualise the discrepancies in predicted $T_{\text {e }}$ across the mountainous terrain of the modelling region (i.e. the interaction between scale, slope, elevation and aspect), we calculated the difference in $\mathrm{T}_{e}$ between the coarse- and finescale model predictions and plotted these differences against slope and elevation (Fig. 2). At low elevations (< approx. $500 \mathrm{~m}$ a.s.l.), the coarse resolution model predicted a lower $\mathrm{T}_{e}$ in comparison to the fine resolution predictions, but with increasing elevation (> approx. $500 \mathrm{~m}$ a.s.l.) the coarse resolution simulation progressively predicted higher operative temperatures (Fig. 2a). Indeed, the greatest discrepancy between the fine and coarse resolution simulations was at an elevation of approximately $1369 \mathrm{~m}$, where the average annual coarse prediction of $\mathrm{T}_{e}$ was $4.3^{\circ} \mathrm{C}$ higher than that of the fine simulation. These discrepancies were typically greatest on steep, as opposed to shallow, slopes, as well as on sites with more northerly (sun-exposed) facing aspects (Fig. 2a).

These differences in $\mathrm{T}_{e}$ among different topographical features and spatial scales in turn led to variation in the insect's predicted TSM. Across this elevation gradient, predicted $\mathrm{T}_{e}$ consistently fell below the insect's optimum temperature for performance (Table 1). The TSM increased with elevation, and sites with shallow, southerly facing slopes had higher TSMs than sites with steep, northerly facing slopes. The effects of slope and elevation on TSM however varied among the different spatial resolutions (Table 1). The coarse resolution estimated a higher average TSM at low elevations than that obtained under the fine-resolution simulations. However once elevation exceeded approximately $500 \mathrm{~m}$ a.s.l. the opposite pattern was observed: insects modelled using a coarse resolution had lower average TSM than that obtained under the fine-resolution simulations (Fig. 2b). Across this elevation transect, discrepancies were more prominent on steep, in comparison to shallow, slopes (Fig. 2b).

Voltinism of the insect varied across the landscape, ranging from one to four generations per year, predictions that correspond with observed phenology of Helicoverpa spp. in the region (K. L. Pringle pers. comm.). Moreover, variation in topography and spatial resolution had direct impacts on insect voltinism, such that the number of generations completed each year declined with increasing elevation (Table 1). The strength of this relationship however, depended on the slope of each site as well as the spatial resolution at which the model was run (Table 1). Indeed, at low elevations the coarse resolution predicted lower voltinism in comparison to the fine scale resolution, while this pattern was reversed at high-elevations (Fig. 2c). The discrepancies in voltinism between spatial resolution was higher on relatively steep slopes (Table 1; Fig. 2c)

\section{Defining the species thermal niche}

The discrepancies in $T_{e}$ predictions between the different spatial resolutions led to differences in size of the predicted thermal niche of the cold and heat sensitive species. For the cold sensitive species the coarse resolution predicted a larger thermal niche (3975 of 4043 cells) than both the fine (3841 of 4043 cells) and medium resolutions (3860 of 4043 cells; Friedman $\chi=237.6, \mathrm{p}<0.001$; Fig. 3a, c, e). In contrast, for the heat sensitive (montane-specialist) species, the fine resolution predicted a larger thermal niche (1150 of 4043 cells) in comparison to the medium (1100 of 4043 cells) and coarse (1005 of 4043 cells) resolutions (Friedman $\chi=136.13$, $\mathrm{p}<0.001$; Fig. 3b, d, f).

\section{Simulating thermoregulatory behaviour within coarse resolution cells}

Allowing the adults of each generation to select the most thermally-optimal conditions for their performance, and thus the conditions encountered by their relatively more immobile offspring, led to significant variation in predicted voltinism and extreme thermal-stress in comparison to simulations in which no thermoregulatory behaviour was allowed (Fig. 4). Allowing adult movement within a coarse grid cell meant that the insect could complete, on average, 0.25 more generations per year $\left(t_{462}=7.698, \mathrm{p}<0.001\right.$; Fig. 4a, b), and avoid, on average, $62.6 \mathrm{~h}$ of extreme cold-stress $\left(t_{464}=-4.63\right.$, $\mathrm{p}<0.001$; Fig. 4c, d). However, laying eggs where $\mathrm{T}_{e}$ was 


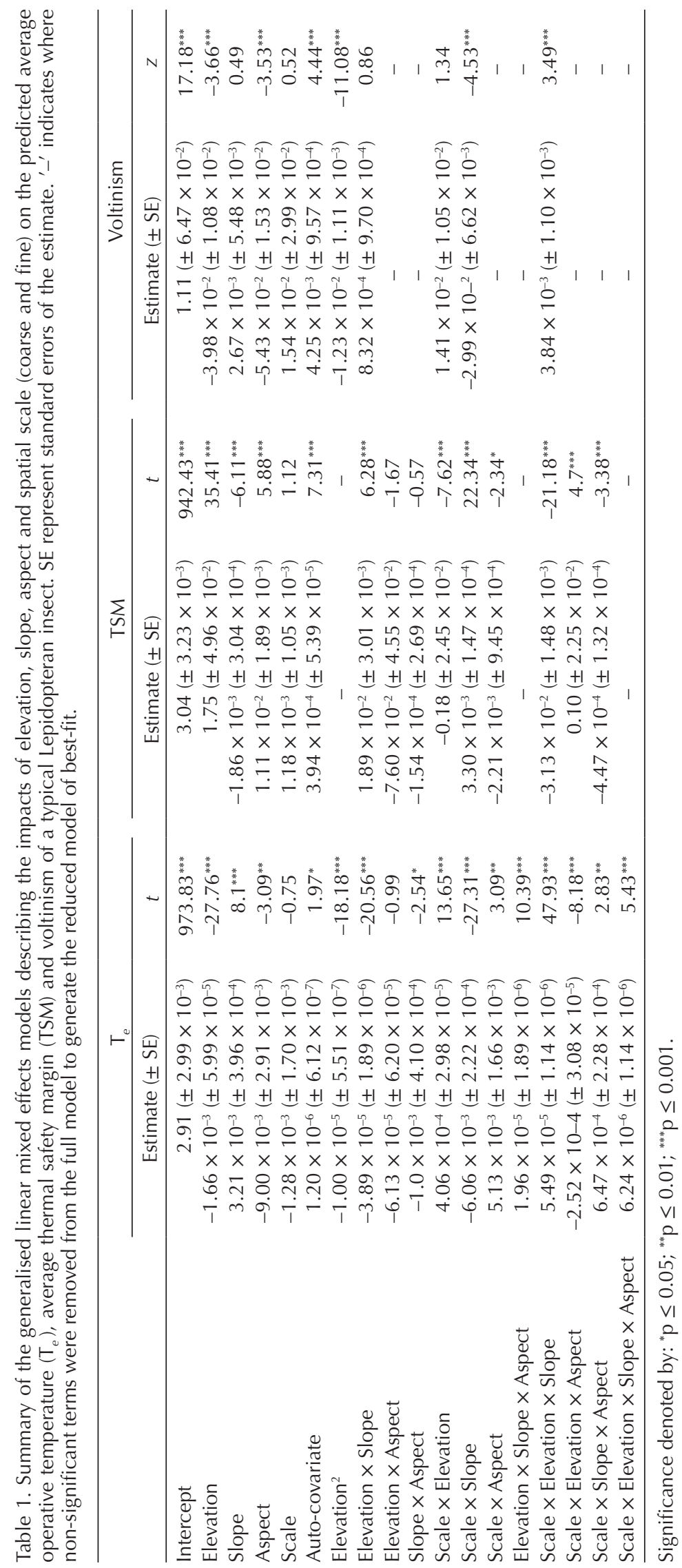



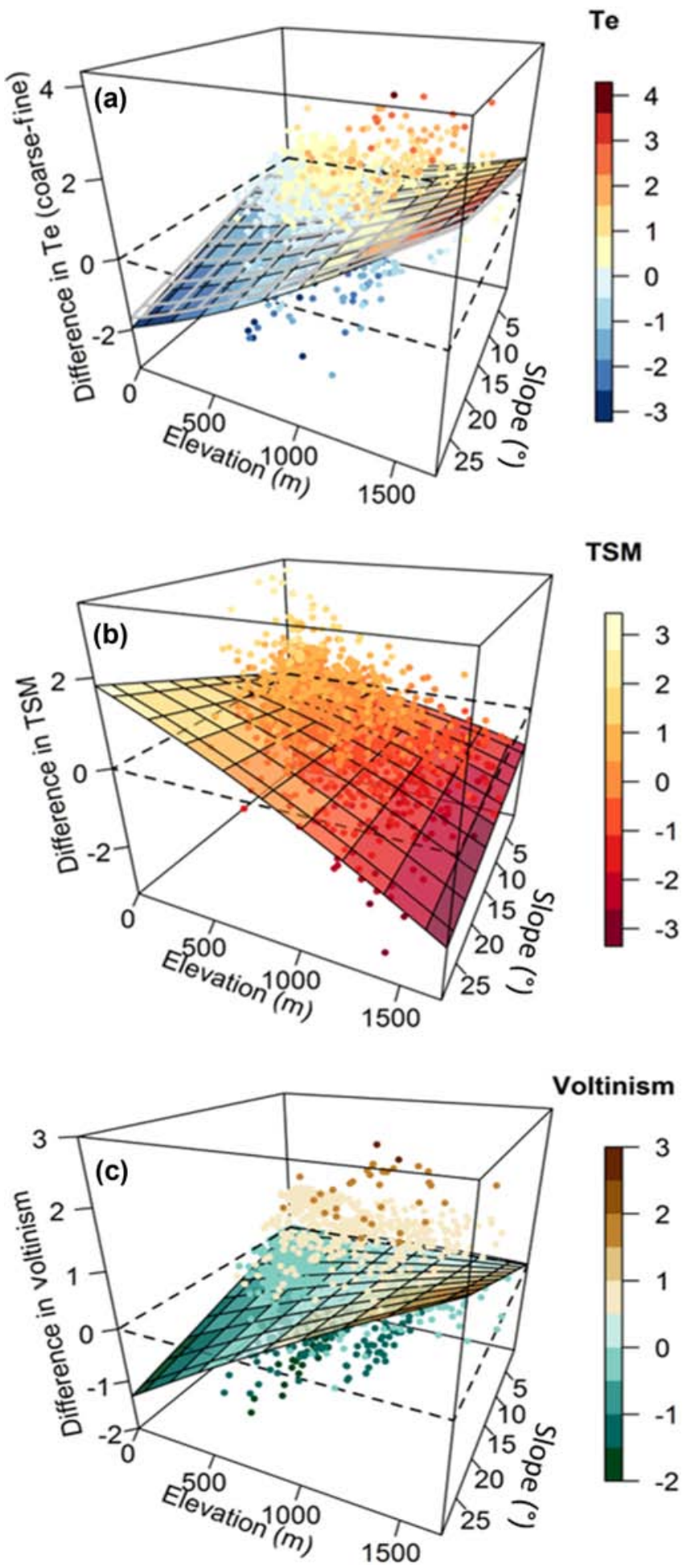

Figure 2. Discrepancies in predicted (a) operative temperature $\left(\mathrm{T}_{e}\right)$, (b) thermal safety margin (TSM) and (c) voltinism between coarse and fine scale model predictions (coarse-fine). Significant interactions were detected between slope, elevation for TSM and voltinism, and also aspect for $\mathrm{T}_{e}$. Surface plots represent predictions of the GLMM regressions, while points show the raw predictions. For $\mathrm{T}_{e}$, coloured surface plot represents discrepancies on sites with northerly aspect, while the surface plot with grey grid lines represent those on southerly facing slopes. Dashed line indicates where there was no difference between coarse and fine simulation predictions. the most optimal for adults resulted in the immature stages experiencing, on average, 1.47 more hours of extreme heatstress in comparison to the 'sessile' organism $\left(t_{464}=-4.45\right.$, $\mathrm{p}<0.001$; Fig. 4e, f).

\section{Discussion}

Using a mechanistic modelling approach we show how variation in topography, combined with differences in the scale used for modelling, affects predictions of $\mathrm{T}_{e}$, and in turn fitness parameters. We also illustrate how model resolution and landscape features interact to influence determination of the potential thermal niche of a typical lepidopteran species. Our results reveal systematic differences in predictions of $\mathrm{T}_{e}$ among models that differ in spatial resolution, largely attributed to variation in local topographic features.

The interactive impacts of elevation, slope, aspect and spatial scale on the discrepancies in $\mathrm{T}_{e}$ appear to have arisen primarily through the associated topographical effects on the calculated solar radiation loads. In our biophysical simulations, an increase in elevation was coupled with an increase in slope, and so at high elevations, calculations of slope between adjacent coarse grid cells are steeper than those between fine grid cells (calculated by the difference in elevation between adjacent cells irrespective of their size; Suggitt et al. 2011, Barton and Terblanche 2014). These apparent steeper slopes in the coarse resolution cells at high elevations in turn led to higher solar radiation loads and warmer predicted $\mathrm{T}_{e}$ (Fig. 2b). In comparison, across 'lowland' topographies fine resolution cells had relatively higher slopes than their coarsecell counterparts (that are averaged across seemingly flat terrain), and thus predicted a higher solar radiation load and $\mathrm{T}_{e}$. When calculated loads of direct and diffuse solar radiation are considered in isolation, it becomes evident that the total solar radiation to which the insect is exposed at high elevations is greater in the coarse-, in comparison to the fine-, resolution simulations, while the opposite is true in lowland regions (Supplementary material Appendix 2 Fig. A2). Overall, such variation in slope among sites is likely to be more accurately represented with fine, rather than coarse, resolution simulations (Scherrer et al. 2011), although we cannot make any inferences here that 'finer is better'. Validations of the model predictions, at both fine and coarse scales, are required to ascertain how accurately this model, as a whole, performs.

An important consideration of our results is to ascertain if the discrepancies in model predictions between the two spatial scales have arisen as a consequence of topographic features specific to the Western Cape region, or if they can be generalized more broadly. For example, the particular shape of the mountain range, which would in turn affect relative changes in slope and aspect across the elevation gradient, might have primarily led to the discrepancies in predictions between the different spatial scales (Elsen and Tingley 2015). To test the generality of our findings we therefore ran some additional simulations (full details shown in Supplementary material Appendix 3), integrating Worldclim datasets of $\mathrm{T}_{\min }, \mathrm{T}_{\max }$, elevation and corresponding slope and aspect at 
two resolutions: a coarse-scale at 10 arc-minutes and a finescale at 5 arc-minutes. Given $H$. armigera is a pest on apple in the Western Cape of South Africa, we focused on other apple growing regions across the globe that cover areas of low and high elevations in: Europe (Fig. 5a), Australia (Fig. 5c), China (Fig. 5e) and the USA (Fig. 5g). Discrepancies in predictions of $\mathrm{T}_{e}$ between the fine- and coarse-scale simulations were similarly detected (Fig. 5). While the magnitude of these discrepancies differed slightly between the regions, all showed a pattern that was consistent with that observed for the Western Cape: in comparison to the finescale resolution, the coarse-scale resolution predicted lower, and then higher $T_{e}$ at low and high elevations respectively (Fig. 5b, d, f, h). These simulations therefore confirm that the patterns detected in this study hold over multiple spatial scales, geographic regions, and mountain shapes.

Discrepancies in the predictions of $\mathrm{T}_{e}$ among the different resolutions led to variation in not only fitness parameters (e.g. voltinism), but also in the potential species' thermal niche based on $\mathrm{T}_{e}$ surpassing critical thermal limits throughout the organism's lifetime. The results are informative for understanding impacts of scale on forecasting efforts for example tropical vs temperate ectotherms (Deutsch et al. 2008, Kearney et al. 2009, Clusella-Trullas et al. 2011). For example, at high elevations the fine-scale simulation predicted colder $\mathrm{T}_{e}$ such that the cold sensitive species appears to be relatively more constrained at this resolution resulting in a smaller thermal niche, in comparison to the coarse- and medium-scale resolution (in which the warmer predicted $\mathrm{T}_{e}$ allows them to persist into higher elevations). By this same process, the heat sensitive species could persist over a broader elevation range (and thus had a larger thermal niche) if the (colder) fine-scale resolution, rather than the (warmer) medium-scale or (hotter) coarse-scale resolutions were considered.

It is important to reiterate here however, we are mapping only the thermal niche (as opposed to the realized niche) of the species - regions where predicted microclimates sustain survival based on thermal suitability (Kearney and Porter 2004). Incorporating additional processes, such as host plant availability (i.e. presence of microclimates and resources), hydro-regulation, rainfall or moisture availability, dispersal barriers and corridors, and species interactions will place further constraints on the species survival and consequently, generate predictions that are closer to the species realised niche, and geographic distribution. Moreover, while the discrepancies in thermal niches among the three scales are relatively small compared to the overall extent of our simulated region

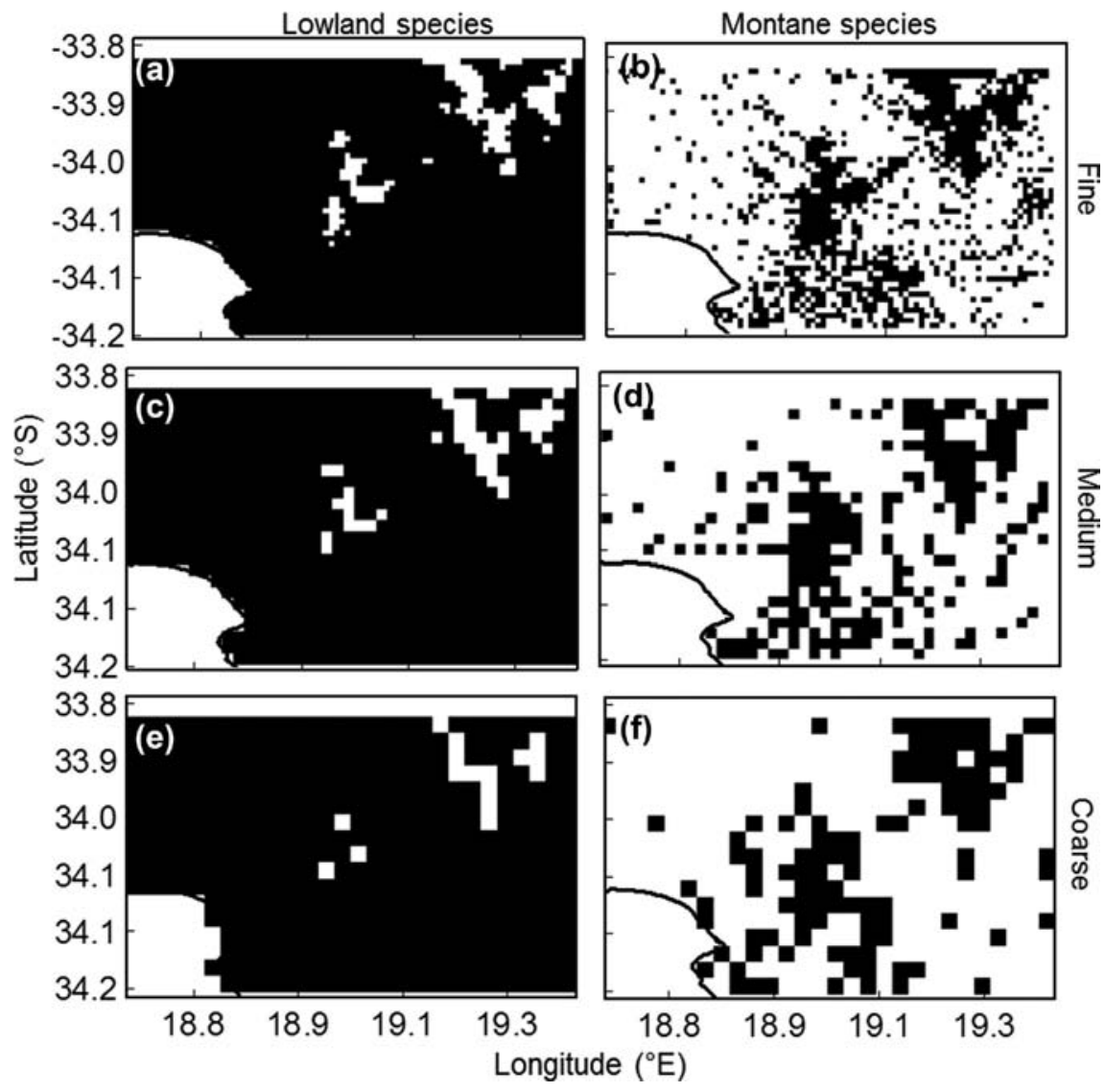

Figure 3. Predictions of the thermal niche for cold ( (a, c, e) 'Lowland') and heat ((b, d, f) 'Montane') sensitive species at fine, medium and coarse grid cell size. Cells with occupancy are shown in black. For the lowland species, predicted thermal niche became progressively smaller as resolution became finer, the opposite pattern occurred for the montane species. 


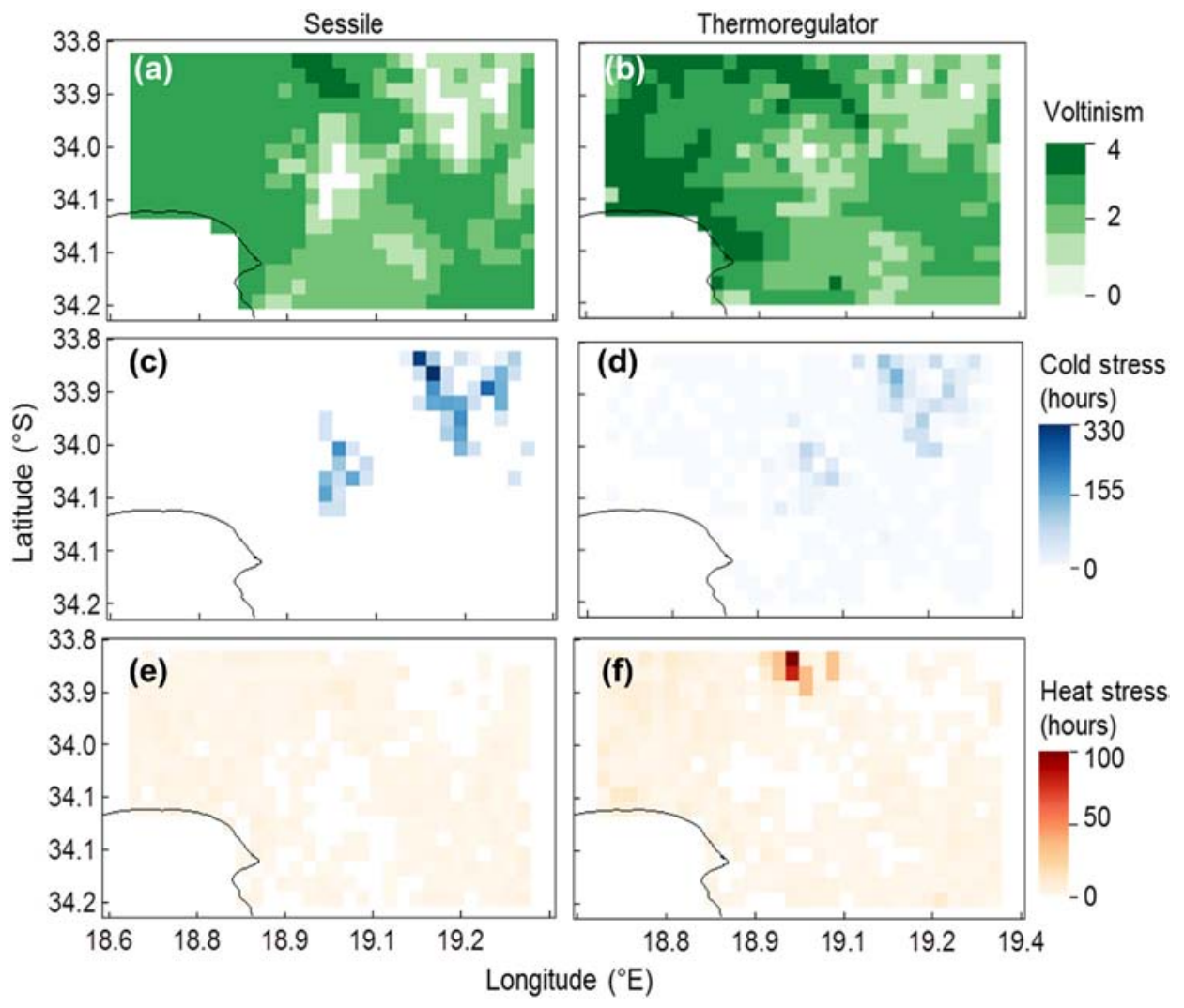

Figure 4. Predicted voltinism, and hours of extreme cold- and heat-stress for sessile (a, c, e) and behaviourally thermoregulating (b, d, f) adults. Overall, the 'thermoregulator' simulation predicted greater voltinism and fewer hours of cold stress, however also encountered a greater number of hours of extreme heat, in comparison to the 'sessile' organism.

(Fig. 3), the modelling approach undertaken here explicitly shows how the spatial resolution of a species' distribution model, whether it be characterizing the fundamental or realized niche, can inherently bias its predictions.

The accuracy of an SDM is likely to be affected not only by its spatial resolution, but also the temporal resolution at which it is run. Across our modelling extent, daily weather data are unfortunately unavailable, and thus we depend on the interpolation of monthly average maximum and minimum thermal conditions into hourly time-steps. While species' distributions may be correlated with such long-term climatic averages, individuals do not typically encounter such conditions in the field, and many studies have shown that SDMs run at high temporal resolutions are more accurate than those run with average long-term climate data (Reside et al. 2010, Sheldon and Dillon 2016). For insects with a holometabolous life-cycle, for which the different life-stages vary in thermal sensitivity and/or developmental requirements, the timing of extreme weather events with respect to the species phenology is likely to a be particularly important determinant of the species distribution (Kingsolver et al. 2011). Incorporating such extreme weather events as part of the input climatic data in the model would likely result in more constrained thermal niches across our modelling extents, though it remains unclear how such constraints would vary among the three spatial resolutions tested here (Buckley and Kingsolver 2012, Buckley and Huey 2016). While running model simulations at different temporal resolutions is beyond the scope of the current study, the modelling framework now developed provides a versatile tool to assess how temporal, as well as spatial, resolution affects the predictions of a species thermal niche.

The interactions between topography and model resolution on $\mathrm{T}_{e}$ can, to some extent explain the discrepancies observed between model resolutions found in previous modelling studies. For example, the future distribution of a Coleoptera species at low elevations declined to a greater extent if models were run at a fine, rather than coarse resolution (according to our findings the fine resolution predicts a higher $\mathrm{T}_{e}$ at low elevations; Gillingham et al. 2012). Similarly, Trivedi et al. (2008) found that at relatively low elevations (between sea level and $1214 \mathrm{~m}$, comparable to our study) modelling species distributions under climate change scenarios led to smaller distributions in fine scale, rather than coarse scale, simulations (also observed in Thomas et al. 2006). In contrast, Randin and colleagues (2009) ran a similar study at higher elevations (ranging from 1480 and $4634 \mathrm{~m}$ ) and found that climate change led to a greater decline in distributions if models were run at coarse, rather than fine resolutions (with the former likely having a higher predicted $\mathrm{T}_{e}$ ). The 

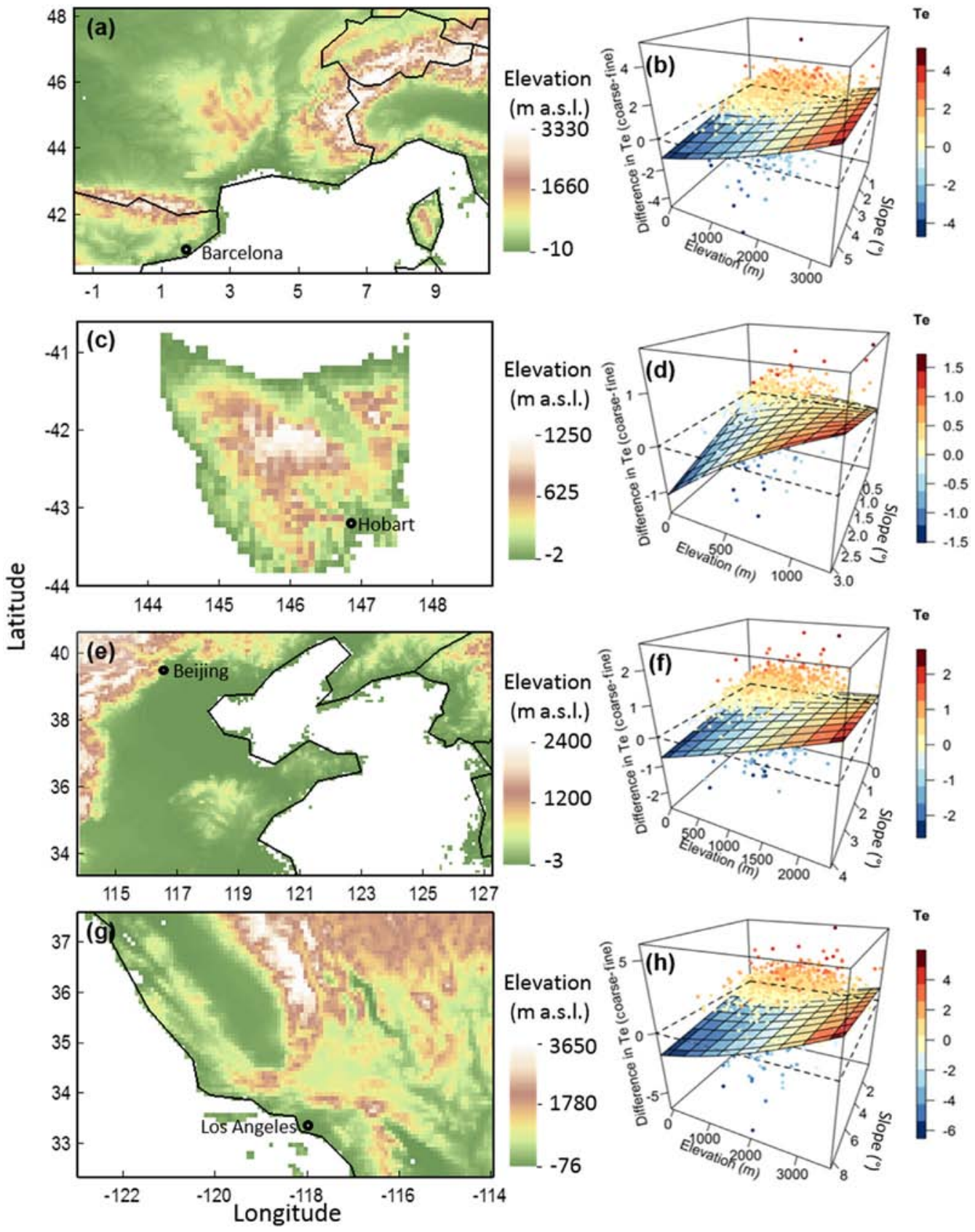

Figure 5. Simulations across four global apple growing regions across which elevation varies, including: (a) Mediterranean Europe; (c) Tasmania, Australia; (e) Shandon, China and (g) California, USA. The largest city in each region is included for reference. Discrepancies in predicted $\mathrm{T}_{\mathrm{e}}$ varied between the coarse and fine-scale resolution model simulations in each region, with lower and then higher predicted $T_{e}$ by the coarse resolution at low and high elevations, respectively $(b, d, f, h)$. Dashed line indicates no difference in $T_{e}$ between coarse and fine predictions.

two latter correlative modelling studies inherently captured the interactions between the model's resolution and topography (with associated variation in solar radiation loads) by including measurements of elevation and solar radiation, and topographically adjusted climate predictors in their models respectively, giving rise to discrepancies in model predictions that are consistent with our findings. These results highlight the importance of accounting for species sensitivity and landscape topography when interpreting the outputs of SDMs, with due consideration given to the mechanism(s) by which such model biases might occur.

While fine-scale resolutions might be required for accurate predictions of sessile organisms such as plants (Trivedi et al. 2008, Randin et al. 2009, Potter et al. 2013), it has been proposed that animals with the capacity to move between grid cells might be accurately modelled at a resolution 
that reflects their home-range size, rather than their body size (see discussions in Kearney et al. 2009, Sears et al. 2011). The topographic variability of the landscape considered here provides a natural mosaic of microclimates across which to test the effects of an animal's behavioural thermoregulation on performance, survival and hence evolutionary fitness (Woods et al. 2015). Indeed, across the Western Cape, thermoregulating insects have an overall increase in the number of generations compared to those that experience the average conditions of the coarse grid cells. The extent to which thermoregulation can improve relative fitness appears to vary with topography: behaviour of individuals at higher elevations had a larger impact on performance than that of individuals at lower elevations, likely due to the greater variation in temperature within each coarse grid cell (unpubl., but see Buckley et al. 2013). Adult thermoregulation also reduced the number of hours of extreme cold-stress, but on the other hand, it simultaneously led to more frequent hours of extreme heat for the offspring. This apparent trade-off highlights the importance of accounting for the life-stage specific sensitives when modelling holometabolous insects, at both spatial and temporal scales (Kingsolver et al. 2011, Kingsolver and Buckley 2015). Quantifying the extent to which adults can optimise conditions for their sessile offspring, as opposed to behaving in a manner that optimizes their own performance as assumed here, warrants further exploration; particularly at resolutions sufficiently fine enough to realistically represent a terrestrial ectotherm's home range (Buckley et al. 2013). The modelling framework developed here provides a sound basis on which to validate such behavioural responses against species of interest.

Our findings highlight that climatic datasets, especially those produced at fine-scales, contain some unavoidable biases (Araújo and Guisan 2006). The Worldclim dataset used here, for example, is interpolated across the landscape using data from isolated weather stations (themselves not uniformly distributed across habitat types), and thus downscaling these conditions into increasingly finer resolutions compounds any inherent biases associated with this procedure (Wilby and Wigley 1997, Tabor and Williams 2010). While further validations of the model here are required, our findings suggest that our understanding of how species thermal niches, and ultimately their distributions, are likely to shift along elevation gradients will at least partly depend on the resolution at which models are run, and inherent issues in the downscaled climatic data are, at least with current global circulation models, unfortunately unavoidable.

Mountainous regions are commonly identified as refuges for terrestrial species, with distributions shifting (often contracting) into higher elevations (Chen et al. 2011, NietoSanchez et al. 2015). These relatively cooler regions, with microclimatic heterogeneity that can perhaps be better exploited by mobile organisms, provide important landforms for the persistence of biodiversity under increasingly stressful climates. Our findings confirm that it is this inherent thermal heterogeneity that makes mountainous regions far more difficult to simulate (i. e. model) than regions with relatively flat terrain. Nonetheless, developing accurate methods to predict how these ecosystems are likely to fare under future climatic and land transformation scenarios is imperative for developing appropriate management and conservation approaches for geographically-restricted or rare species. This is also the case for managers dealing with invasive species, agricultural pests and disease vectors. Here, we have shown systematic differences in how spatial scale can affect model predictions with respect to elevation, differences that should be accounted for when interpreting predictions of species distribution models.

Acknowledgements - We are grateful for insightful and constructive comments on an earlier version of this work by Peter le Roux, Raquel Garcia and the anonymous reviewers.

Funding - Funding was provided by HORTRGRO Science and JST and SCT were supported by separate funding from the National Research Foundation Incentive Funding for Rated (NRF IFR) researchers. MGB was supported by the Claude Leon Foundation. Author contributions - MGB, JST and SCT conceived and designed methodology. MGB ran model simulations, analysed data and led writing of the manuscript. All authors contributed significantly to multiple drafts of the manuscript and gave final approval for its publication.

\section{References}

Acquah, H. D. 2010. Comparison of Akaike information criterion (AIC) and Bayesian information criterion (BIC) in selection of an asymmetric price relationship. - J. Develop. Agric. Econ. 2: $1-6$.

Araújo, M. B. and Guisan, A. 2006. Five (or so) challenges for species distribution modelling. - J. Biogeogr. 33: 1677-1688.

Austin, M. P. and Van Niel, K. P. 2010. Improving species distribution models for climate change studies: variable selection and scale. - J. Biogeogr. 38: 1-8.

Bakken, G. S. 1992. Measurement and application of operative and standard operative temperatures in ecology. - Am. Zool. 32: $194-216$.

Barton, K. 2016. MuMIn: multi-model inference. - R package ver. 1.15.6.

Barton, M. G. and Terblanche, J. S. 2014. Predicting performance and survival across topographically heterogeneous landscapes: the global pest insect Helicoverpa armigera (Lepidoptera: Noctuidae). - Aust. Entomol. 53: 249-258.

Basson, C. H. et al. 2017. Lizards paid a greater opportunity cost to thermoregulate in a less heterogeneous environment. - Funct. Ecol. 31: 856-865.

Beck, J. et al. 2014. Spatial bias in the GBIF database and its effect on modeling species' geographic distributions. - Ecol. Inform. 19: 10-15.

Bennie, J. et al. 2008. Slope, aspect and climate: spatially explicit and implicit models of topographic microclimate in chalk grassland. - Ecol. Model. 216: 47-59.

Billings, W. D. 1973. Arctic and alpine vegetation: similarities, differences, and susceptibility to disturbance. - BioScience 23: 697-704. 
Bivand, R. and Piras, G. 2015. Comparing implementations of estimation methods for spatial econometrics. - J. Stat. Softw. 63: $1-36$.

Bonebrake, T. C. et al. 2010. Oviposition behavior and offspring performance in herbivorous insects: consequences of climatic and habitat heterogeneity. - Oikos 119: 927-934.

Bonebrake, T. C. et al. 2014. From global change to a butterfly flapping: biophysics and behaviour affect tropical climate change impacts. - Proc. R. Soc. B 281: 20141264.

Buckley, L. B. and Kingsolver, J. G. 2012. The demographic impacts of shifts in climate means and extremes on alpine butterflies. - Funct. Ecol. 26: 969-977.

Buckley, L. B. and Huey, R. B. 2016. How extreme temperatures impact organisms and the evolution of their thermal tolerance. - Integr. Comp. Biol. 56: 98-109.

Buckley, L. B. et al. 2010. Can mechanism inform species distribution models? - Ecol. Lett. 13: 1041-1054.

Buckley, L. B. et al. 2013. Can terrestrial ectotherms escape the heat of climate change by moving? - Proc. R. Soc. B 280: 20131149.

Buckley, L. B. et al. 2015. Thermoregulatory behaviour limits local adaptation of thermal niches and confers sensitivity to climate change. - Funct. Ecol. 29: 1038-1047.

Campbell, G. S. and Norman, J. M. 1998. An introduction of environmental biophysics, 2 nd ed. - Springer Science.

Chen, I. C. et al. 2011. Rapid range shifts of species associated with high levels of climate warming. - Science 333: 1024-1026.

Clusella-Trullas, S. et al. 2011. Climatic predictors of temperature performance curve parameters in ectotherms imply complex responses to climate change. - Am. Nat. 177: 738-751.

Deutsch, C. A. et al. 2008. Impacts of climate warming on terrestrial ectotherms across latitude. - Proc. Natl Acad. Sci. USA 105: 6668-6672.

Dormann, C. F. et al. 2007. Methods to account for spatial autocorrelation in the analysis of species distributional data: a review. - Ecography 30: 609-628.

Elith, J. and Leathwick, J. R. 2009. Species distribution models: ecological explanation and prediction across space and time. - Annu. Rev. Ecol. Evol. Syst. 40: 677-697.

Elsen, P. R. and Tingley, M. W. 2015. Global mountain topography and the fate of montane species under climate change. - Nat. Clim. Change 5: 772-776.

Flint, L. E. and Flint, A. L. 2012. Downscaling future climate scenarios to fine scales for hydrologic and ecological modeling and analysis. - Ecol. Process. 1: 1-15.

Franklin, J. et al. 2013. Modeling plant species distributions under future climates: how fine scale do climate projections need to be? - Global Change Biol. 19: 473-483.

Gillingham, P. K. et al. 2012. The effect of spatial resolution on projected responses to climate warming. - Divers. Distrib. 18: 990-1000.

Gräler, B. et al. 2016. Spatio-temporal interpolation using gstat. - RFID J. 8: 204-218.

Guisan, A. and Thuiller, W. 2005. Predicting species distribution: offering more than simple habitat models. - Ecol. Lett. 8: 993-1009.

Guisan, A. et al. 2007. Sensitivity of predictive species distribution models to change in grain size. - Divers. Distrib. 13: 332-340.

Hannah, L. et al. 2014. Fine-grain modeling of species' response to climate change: holdouts, stepping-stones, and microrefugia. - Trends Ecol. Evol. 29: 390-397.
Helmuth, B. et al. 2006. Living on the edge of two changing worlds: forecasting the responses of rocky intertidal ecosystems to climate change. - Annu. Rev. Ecol. Evol. Syst. 37: 373-404.

Hertz, P. E. et al. 1993. Evaluating temperature regulation by field-active ectotherms: the fallacy of the inappropriate question. - Am. Nat. 142: 796-818.

Hijmans, R. J. and van Etten, J. 2012. Raster: geographic analysis and modeling with raster data. - $\mathrm{R}$ package ver. 2.0-12.

Hijmans, R. J. et al. 2005. Very high resolution interpolated climate surfaces for global land areas. - Int. J. Climatol. 25: 1965-1978.

Kearney, M. and Porter, W. P. 2004. Mapping the fundamental niche: physiology, climate, and the distribution of a nocturnal lizard. - Ecology 85: 3119-3131.

Kearney, M. et al. 2009. The potential for behavioural thermoregulation to buffer 'cold-blooded' animals against climate warming. - Proc. Natl Acad. Sci. USA 106: 3835-3840.

King, A. B. et al. 1990. A mark-capture study of Helicoverpa armigera dispersal from pigeon-pea in southern India. - Entomol. Exp. Appl. 55: 257-266.

Kingsolver, J. G. and Buckley, L. B. 2015. Climate variability slows evolutionary responses of Colias butterflies to recent climate change. - Proc. R. Soc. B 282: 20142470.

Kingsolver, J. G. et al. 2011. Complex life cycles and the responses of insects to climate change. - Integr. Comp. Biol. 51: 719-732.

Lawson, C. R. et al. 2014. Topographic microclimates drive microhabitat associations at the range margin of a butterfly. - Ecography 37: 732-740.

Levy, O. et al. 2014. Approaches to advance scientific understanding of macrosystems ecology. - Front. Ecol. Environ. 12: 15-23.

Luoto, M. and Heikkinen, R. K. 2008. Disregarding topographical heterogeneity biases species turnover assessments based on bioclimatic models. - Global Change Biol. 14: 483-494.

Mironidis, G. K. 2014. Development, survivorship and reproduction of Helicoverpa armigera (Lepidoptera: Noctuidae) under fluctuating temperatures. - Bull. Entomol. Res. 104: 751-764.

Mironidis, G. K. and Savopoulou-Soultani, M. 2008. Development, survivorship, and reproduction of Helicoverpa armigera (Lepidoptera: Noctuidae) under constant and alternating temperatures. - Environ. Entomol. Am. 37: 16-28.

Nadeau, C. P. et al. 2017. Coarse climate change projections for species living in a fine-scaled world. - Global Change Biol. 23: 12-24.

Neter, J. et al. 1996. Applied linear statistical models. - Irwin.

Nieto-Sanchez, S. et al. 2015. Long-term change and spatial variation in butterfly communities over an elevational gradient: driven by climate, buffered by habitat. - Divers. Distrib. 21: 950-961.

Parmesan, C. and Yohe, G. 2003. A globally coherent fingerprint of climate change impacts across natural systems. - Nature 42 : $37-42$.

Pinheiro, J. et al. 2016. nlme: linear and nonlinear mixed effects models. - R package ver. 3.1-128.

Potter, K. A. et al. 2013. Microclimatic challenges in global change biology. - Global Change Biol. 19: 2932-2939.

Randin, C. F. et al. 2009. Climate change and plant distribution: local models predict high-elevation persistence. - Global Change Biol. 15: 1557-1569.

Reside, A. E. et al. 2010. Weather, not climate, defines distributions of vagile bird species. - PLoS One 5: e13569. 
Rosenheim, J. A. et al. 2008. Time limitation, egg limitation, the cost of oviposition, and lifetime reproduction by an insect in nature. - Am. Nat. 172: 486-496.

Ruiz-Aravena, M. et al. 2014. Impact of global warming at the range margins: phenotypic plasticity and behavioural thermoregulation will buffer an endemic amphibian. - Ecol. Evol. 4: 4467-4475.

Scherrer, D. and Körner, C. 2011. Topographically controlled thermal-habitat differentiation buffers alpine plant diversity against climate warming. - J. Biogeogr. 38: 406-416.

Scherrer, D. et al. 2011. Elevational species shifts in a warmer climate are overestimated when based on weather station data. - Int. J. Biometeorol. 55: 645-654.

Schulze, R. E. 1997. South African atlas of agro-hydrology and climatology. - Report TT 82/96, Water Research Commission, Pretoria.

Sears, M. W. et al. 2011. The world is not flat: defining relevant thermal landscapes in the context of climate change. - Integr. Comp. Biol. 51: 666-675.

Sears, M. W. et al. 2016. Configuration of the thermal landscape determines thermoregulatory performance of ectotherms. - Proc. Natl Acad. Sci. USA 113: 10595-10600.

Seo, C. et al. 2009. Scale effects in species distribution models: implications for conservation planning under climate change. - Biol. Lett. 5: 39-43.

Sheldon, K. S. and Dillon, M. E. 2016. Beyond the mean: biological impacts of cryptic temperature change. - Integr. Comp. Biol. 56: 110-119.

Slavich, E. et al. 2014. Topoclimate versus macroclimate: how does climate mapping methodology affect species distribution models and climate change projections? - Divers. Distrib. 20: 952-963.

Supplementary material (Appendix ECOG-03655 at <www. ecography.org/appendix/ecog-03655>). Appendix 1-3.
Stevenson, R. D. 1985. The relative importance of behavioural and physiological adjustments controlling body temperature in terrestrial ectotherms. - Am. Nat. 126: 362-386.

Suggitt, A. J. et al. 2011. Habitat microclimates drive fine-scale variation in extreme temperatures. - Oikos 120: 1-8.

Sunday, J. M. et al. 2014. Thermal-safety margins and the necessity of thermoregulatory behavior across latitude and elevation. - Proc. Natl Acad. Sci. USA 111: 5610-5615.

Tabor, K. and Williams, J. W. 2010. Globally downscaled climate projections for assessing the conservation impacts of climate change. - Ecol. Appl. 20: 554-565.

Thomas, C. D. et al. 2006. Range retractions in the face of climate warming. - Trends Ecol. Evol. 21: 415-416.

Trivedi, M. R. et al. 2008. Spatial scale affects bioclimate model projections of climate change impacts on mountain plants. - Global Change Biol. 14: 1089-1103.

Van doninck, J. 2016. horizon: Horizon Search Algorithm. - $\mathrm{R}$ package ver. 1.0 .

Weiss, S. B. et al. 1988. Sun, slope, and butterflies; topographic determinants of habitat quality for Euphydryas editha. - Ecology 69: 1486-1896.

Wiens, J. J. et al. 2010. Niche conservatism as an emerging principle in ecology and conservation biology. - Ecol. Lett. 13: 1310-1324.

Wilby, R. L. and Wigley, T. M. L. 1997. Downscaling general circulation model output: a review of methods and limitations. - Prog. Phys. Geogr. 21: 530-548.

Woods, H. A. et al. 2015. The roles of microclimatic diversity and of behaviour in mediating the responses of ectotherms to climate change. - J. Therm. Biol. 54: 86-97.

Zuur, A. F. et al. 2009. Mixed effects models and extensions in ecology with R. - Springer. 